Gamonales, J.M.; Muñoz-Jiménez, J.; León, K.; Ibáñez, S.J. (2019). Effectiveness of the Launch at FA5 for Blind Persons in 2016 Paralympic Games. Revista Internacional de Medicina y $\begin{array}{lllllll}\text { Ciencias de la Actividad Física y el Deporte vol. } 19 & \text { (76) pp. } & 745-764\end{array}$ Http://cdeporte.rediris.es/revista/revista76/arteficacia1099.htm

DOI: $10.15366 /$ rimcafd2019.76.012

\title{
ORIGINAL
}

\section{EFICACIA DEL LANZAMIENTO EN FA5 PARA PERSONAS CIEGAS EN LOS JUEGOS PARALÍMPICOS DE 2016}

\section{EFFECTIVENESS OF THE LAUNCH AT FA5 FOR BLIND PERSONS IN 2016 PARALYMPIC GAMES}

\author{
Gamonales, J.M. ${ }^{\text {; }}$ Muñoz-Jiménez, J. ${ }^{1,2}$; León, K. ${ }^{1}$ e Ibáñez, S.J.1 \\ ${ }^{1}$ Facultad de Ciencias de la Actividad Física y el Deporte. Universidad de Extremadura \\ (España)martingamonales@unex.es, suliwan@unex.es, fleon@unex.es, sibanez@unex.es \\ 2 Universidad Autónoma de Chile (Chile) jesus.munoz@uautonoma.cl
}

\section{AGRADECIMIENTOS Y/O FINANCIACIÓN}

Trabajo desarrollado dentro del Grupo de Optimización del Entrenamiento y Rendimiento Deportivo (G.O.E.R.D.) de la Facultad de Ciencias del Deporte de la Universidad de Extremadura.

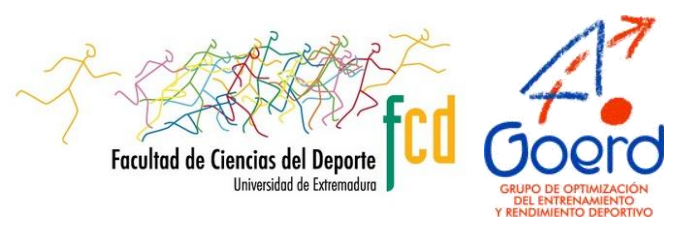

Este trabajo ha sido parcialmente subvencionado por la Ayuda a los Grupos de Investigación (GR18170) de la Junta de Extremadura (Consejería de Empleo e Infraestructuras); con la aportación de la Unión Europea a través de los Fondos Europeos de Desarrollo Regional (FEDER).

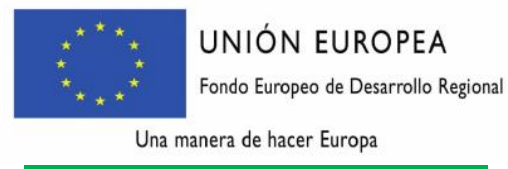

\section{JUNTA DE EXTREMADURA}

Código UNESCO / UNESCO code: 5899 Educación Física y Deportiva / Physical Education and Sports.

Clasificación Consejo de Europa / Council of Europe classification: 17. Otras: Procesos de percepción / Others: Perception processes.

Recibido 13 de febrero de 2018 Received February 13, 2018

Aceptado 12 de julio de 2019 Accepted July 12, 2019 


\section{RESUMEN}

El presente estudio analiza todos los lanzamientos a portería $(n=730)$ en la modalidad Fútbol a 5 para ciegos de los Juegos Paralímpicos de 2016. Para ello, se utilizó el instrumento IOLF5C para analizar la eficacia del lanzamiento en Fútbol a 5 para personas ciegas, que emplea 13 variables para definir cada lanzamiento. Se realizó un estudio descriptivo, y tras analizar las relaciones entre las variables y la eficacia, se identificó el tipo de lanzamiento más eficaz, así como sus asociaciones significativas. El Análisis de Regresión Logística muestra la existencia de tres variables que predicen la eficacia del lanzamiento a portería en relación a la Zona de comienzo y Tipo de golpeo. Estos resultados permiten caracterizar el Fútbol a 5 para personas ciegas y lo diferencia del resto de deportes de tanteo bajo en los tipos de progresión, bloqueos y modos de juego.

PALABRAS CLAVE: fútbol, lanzamiento, eficacia, Juegos Paralímpicos.

\section{ABSTRACT}

The present study analysed all shots at goal $(n=730)$ in football 5 -a-side for the blind at the 2016 Paralympic Games. The instrument IOLF5C was used to analyse shooting effectiveness in football 5 -a-side for the blind. It comprises 13 variables that define every shot. A descriptive study was carried out, and after analysing the significant relationships between all variables and effectiveness, the most effective type of shot was identified, as well as the most significant associations. The logistic regression analysis revealed the existence of two variables that predict shooting effectiveness: starting zone and type of contact. These results allowed for characterisation of football 5-a-side for the blind and its differentiation from other low-scoring sports regarding types of advancement, blocks and playing systems.

KEY WORDS: football, launching, effectivenes, Paralympic Games.

\section{INTRODUCCIÓN}

El Fútbol a 5 para personas ciegas y con discapacidad visual (en adelante Fa5) es un deporte de cooperación oposición (Hernández, 2005) practicado al aire libre para permitir una acústica óptima para los deportistas. Se juega entre dos equipos de cinco jugadores cada uno (un portero y cuatro jugadores de campo) y se ha convertido en uno de los deportes más populares entre este colectivo. Los trabajos relacionados con el Fa5 no son frecuentes en la literatura (Gamonales, 2017). Se pueden encontrar trabajos que analizan las estrategias de los jugadores con respecto a la lectura del juego en Fa5 (Morato, Gomes, Duarte \& De Almeida, 2011), que estudian los efectos del entrenamiento en los parámetros de la aptitud física y la composición corporal (Campos et al., 2013) o las alteraciones cardiorrespiratorias y el perfil motor de los jugadores de Fa5 de selecciones (Campos et al., 2014). Otros trabajos se centran en evaluar las características y la prevalencia de las lesiones relacionadas con los deportes de personas con discapacidad visual, incluyendo el Fa5 (Magno, Morato, Bilzon \& Duarte, 2013). Además, hay estudios que comparan la relación existente entre 
la representación mental y las direcciones de sonido entre los futbolistas ciegos, no atletas ciegos y personas con visión (Velten, Bläsing, Portes, Hermann \& Schack, 2014; Velten, Ugrinowitsch, Portes, Hermann \& Bläsing, 2016). Por otro lado, Suarez (2014), analiza el trabajo del guía en los jugadores de fútbol para ciegos y, por último, Gorla et al. (2017) determinan la composición corporal y somatotipo perfil de los jugadores de la selección brasileña de Fa5. Sin embargo, no hay ningún trabajo que analice el rendimiento deportivo del Fa5 de manera específica.

El análisis del rendimiento deportivo es una de las líneas de investigación más relevantes de las Ciencias del Entrenamiento. Se trata de una línea emergente, que hace relativamente poco tiempo ha ganado popularidad como un término entre los investigadores y profesionales del deporte (Drust, 2010). El análisis científico del rendimiento deportivo tiene como objetivo avanzar en el conocimiento del contexto de juego con vistas a mejorar los resultados futuros (McGarry, 2009). Para Hughes \& Bartlett (2002), dentro del análisis del rendimiento deportivo se incluyen todas las investigaciones que analizan el rendimiento deportivo en competición real o durante el entrenamiento. Pero lo que lo distingue de otras disciplinas de las Ciencias del Deporte, es el interés por el rendimiento deportivo real durante la competición más que los conocimientos derivados de los informes sobre los deportistas, de las pruebas de laboratorio 0 de la información obtenida de los deportistas a través de cuestionarios, grupos de discusión y/o entrevistas (O'Donoghue, 2010). Es decir, tiene su propio espacio en las Ciencias del Deporte con unas características de elevada aplicación al entrenamiento y el control de la competición (Gómez-Ruano, 2017), buscando conocer cómo predecir y prescribir el rendimiento de jugadores y equipos sobre la base de las características de la propia competición (O'Donoghue, 2015; Rein \& Memmert, 2016).

Los indicadores de rendimiento son una selección o combinación de variables de movimiento que tiene el objetivo de definir algunos, o todos, los aspectos del rendimiento de un contexto deportivo. Analistas y entrenadores usan los indicadores de rendimiento para medir el rendimiento individual, de diferentes elementos del grupo o de un equipo (Hughes \& Bartlett, 2002).

La acción más estudiada, considerada como factor e indicador de máxima eficacia, en el juego es el lanzamiento o remate (Ibáñez, Feu, García-Rubio, Parejo \& Cañadas, 2009) porque es la actividad que condiciona el éxito deportivo. Pero en trabajos específicos de análisis de rendimiento también ha sido analizada la zona del lanzamiento (Reina-Gómez, Hernández-Mendo \& Fernández-García, 2010), el rol de los jugadores (Tsitskaris, Theoharopoulos, Galanis \& Nikopoulou, 2002), la presión defensiva y el nivel de los jugadores (Ibáñez, Santos \& García-Rubio, 2015) así como las acciones en situación de ataque (Álvarez, Puente, Manero \& Manonelles, 2004; Lago-Peñas; MartínAcero \& Seirul-lo, 2007; Valez, Areces, Blanco \& Arce, 2011). También se encuentran estudios sobre el momento de marcar el gol (García-Rubio, GómezRuano, Lago-Peñas, \& Ibáñez, 2017; Lago-Peñas, Lago-Ballestero, Dellal \& Gómez-Ruano, 2010; Liu, Gómez-Ruano, Lago-Peñas \& Sampaio, 2015), número de lanzamientos a portería (Tenga, Ronglan \& Bahr, 2010), localización del partido (Caballero, García-Rubio \& Ibáñez, 2017), así como la situación del 
equipo cuando gana, empata o pierde (Castellano, Casamichana \& Lago-Peñas, 2012; García-Rubio, Ibáñez, Gómez-Ruano \& Sampaio, 2014; Gómez-Ruano, Gómez-López \& Jiménez Sáiz, 2013; Hughes \& Franks, 2005).

Los indicadores de rendimiento aplicados en los deportes colectivos permiten conocer la lógica del juego a través de los factores técnico-tácticos (GómezRuano, 2017; Reina-Gómez \& Hernández-Mendo, 2012). Es decir, se describen variables concretas, ofensivas o defensivas (Thomas, Fellingham \& Vehrs, 2009; Ibáñez et al., 2009; Marcelino, Mesquita \& Sampaio, 2011; Sampaio, LagoPeñas, Casais \& Leite, 2010), permitiendo a los entrenadores un mejor control de los entrenamientos y la competición (Gómez-Ruano, Ibáñez, Parejo \& Furley, 2017).

Para conocer la modalidad objeto del estudio, el Fa5 para personas ciegas 0 discapacidad visual, habrá que tener en cuenta estudios de indicadores de rendimiento así como todas las variables relacionadas con el lanzamiento a portería específicas del Fa5. Por ello, los objetivos generales de esta investigación fueron: i) analizar los lanzamientos a portería de los Juegos Paralímpicos de 2016, en la modalidad de Fa5 ii) conocer las relaciones que condicionan la eficacia del lanzamiento a portería, y por último, iii) predecir la eficacia del lanzamiento. Este artículo se encuadra en los estudios sobre el Proceso de juego para aumentar el conocimiento técnico-táctico (Ibáñez, Sampaio, Sáenz-López, Giménez \& Janeira, 2003) en la modalidad Fa5, y contribuye a aumentar el interés de los clubes, deportistas, medios de comunicación, entrenadores o aficionados por conocer cómo rinden los jugadores y la explicación que se puede dar a su rendimiento tanto en competición como en entrenamiento (O`Donoghue, 2015; Gómez-Ruano, 2017).

\section{MÉTODO}

\section{Diseño}

El diseño utilizado en esta investigación se posiciona dentro de los estudios empíricos cuantitativos, mediante un código arbitrario de observación, realizado en un contexto natural (Montero \& León, 2007).

\section{Muestra}

Se analizaron todos los lanzamientos en juego $(n=730)$ realizados en los partidos $(n=18)$ de Fa5 celebrado en Brasil 2016 de los Juegos Paralímpicos.

\section{Variables}

La definición de cada variable del estudio y sus categorías se plantearon en un estudio previo con un grupo de expertos, configurado por entrenadores de nivel nacional e internacional de Fa5, y se definió de forma precisa cuáles eran las susceptibles de ser observadas (Gamonales, León, Muñoz, González-Espinosa \& Ibáñez, 2018a). Concretamente, se incluyó la totalidad de las variables contextuales, de juego y de resultado que constituyen el bloque I del instrumento 
IOLF5C, diseñado y validado para conocer los Indicadores de Rendimiento Competitivo en Fútbol a 5 para personas ciegas (Gamonales, Muñoz, León \& Ibáñez, 2018b). Dichas variables serán usadas para el análisis de los indicadores de rendimiento (O'Donoghue, 2010). Todas se categorizaron de forma numérica, con la finalidad de facilitar su registro y posterior análisis estadístico. Las variables registradas fueron:

Variables contextuales: Fase, Tiempo de los lanzamientos, Situación lanzador, Resultado final.

Variables de juego: Zona de comienzo, Tipo de progresión, Zona de lanzamiento, Situación de golpeo, Bloqueo, Oposición en el lanzamiento, Zona corporal, Tipo de golpeo.

Variable de resultado: Resultado del lanzamiento.

Para el análisis predictivo, se realizó un proceso de recodificación de la variable Resultado del lanzamiento, y la variable se definió como Resultado Recodificado.

\section{Procedimiento}

Para analizar la eficacia del lanzamiento en Fa5 se utilizó el instrumento IOLF5C (Gamonales et al., 2018a). Para ello, se empleó un proceso de entrenamiento de codificadores con el fin de confirmar si los datos son válidos y fiables pondrán ser empleados para la investigación. El entrenamiento de los codificadores estuvo dividido en cuatro etapas: etapa preparatoria, etapa de selección de los codificadores, etapa de formación de los observadores, y, por último, etapa de confiabilidad con un valor de Kappa de 0,95 (Gamonales et al., 2018b). Tras la formación de los codificadores, estos registraron todos los lanzamientos del campeonato $(n=730)$ de forma individual, lo que permitió obtener todos los registros para realizar el pertinente análisis estadístico.

\section{Análisis estadístico}

Se realizó un análisis descriptivo de frecuencias y porcentajes. Para estimar la asociación entre las variables, se empleó Chi cuadrado $\left(x^{2}\right)$ y Coeficiente Phi $(\varphi c)$ de Cramer (Newell, Aitchison \& Grant, 2014). El nivel de asociación del indicador $\varphi c$ de Cramer se interpreta a través de la propuesta de Crewson (2006). El grado de asociación entre las variables se analizó a través de los Residuos Tipificados Corregidos (RTC) de las tablas de contingencia (Field, 2009), resultantes de relacionar las variables independientes del estudio (contextuales y acciones de juego) con la variable del estudio (Resultado del lanzamiento).

Para determinar la Eficacia, se realizó un proceso de recodificación de la variable Resultado del lanzamiento para el Modelo de Regresión, agrupando las categorías para orientar el análisis de forma específica. Las variables independientes incluidas en el modelo fueron seleccionadas porque mostraron una relación estadísticamente significativa en la prueba Chi cuadrado $\left(X^{2}\right)$ del 
Análisis Inferencial. Tras la recodificación de las categorías de estas variables, en función de las frecuencias más altas del Análisis Descriptivo Univariado fueron introducidas en el sistema, pues no existen investigaciones previas en este contexto deportivo en las que basar el contraste de hipótesis (Agresti \& Finlay, 2009; Field, 2009).

Los resultados del análisis predictivo tienen por finalidad valorar si existe una función estadísticamente significativa que relacione las variables independientes definidas para el estudio con la variable dependiente que define la eficacia, al igual que en estudios previos de análisis de juego en fútbol (García-Rubio et al., 2017), u otras modalidades deportivas de equipo (Gómez-Ruano et al., 2017; lbáñez et al., 2009). Las variables que se incluyeron como candidatas en el análisis de Regresión Logística Binaria, fueron Zona de comienzo y Tipo de golpeo. El análisis de Regresión Logística utilizó dos pasos para establecer el modelo de predicción, dando como resultado la inclusión de las dos variables en el modelo final. El método utilizado fue la Regresión Logística por pasos o "Stepwise" hacía delante basado en el estadístico de Wald. El punto de corte para la clasificación de los casos, se estableció a través de la técnica de la Curva ROC (Receiver Operating Characteristic o Característica Operativa del Receptor). Por último, se evaluó el ajuste de los datos al modelo de Regresión Logística a través de la prueba de bondad de ajuste de Hosmer-Lemeshow y la matriz de correlación de las variables incluidas en el modelo final de regresión (Agresti \& Finlay, 2009; Field, 2009; Menard, 2002). El análisis de datos fue realizado usando el paquete estadístico SPSS 24.0 (IBM SPSS Statistics 24.0, Chicago, IL, USA).

\section{RESULTADOS}

En la figura 1, se muestran los lanzamientos que realizaron los equipos según las fases establecidas en los Juegos Paralímpicos 2016, en la modalidad deportiva de fútbol a 5 para personas ciegas.

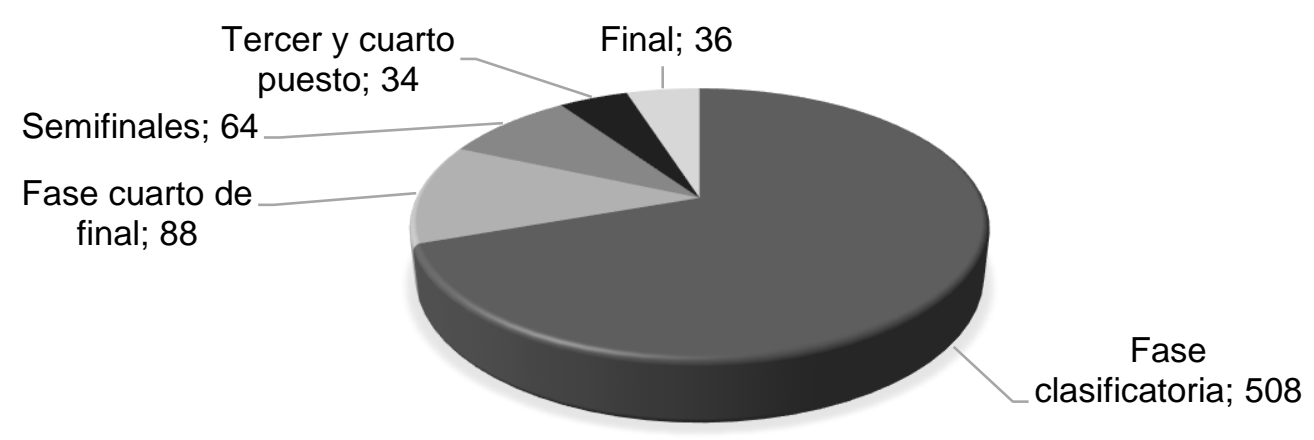

Figura 1. Lanzamientos a portería realizados en las distintas fases de los Juegos Paralímpicos de Fa5 del 2016.

En la figura 2, se muestran las franjas de tiempo en las que se realizaron los lanzamientos a portería. 
Rev.int.med.cienc.act.fís.deporte - vol. 19 - número 76 - ISSN: 1577-0354

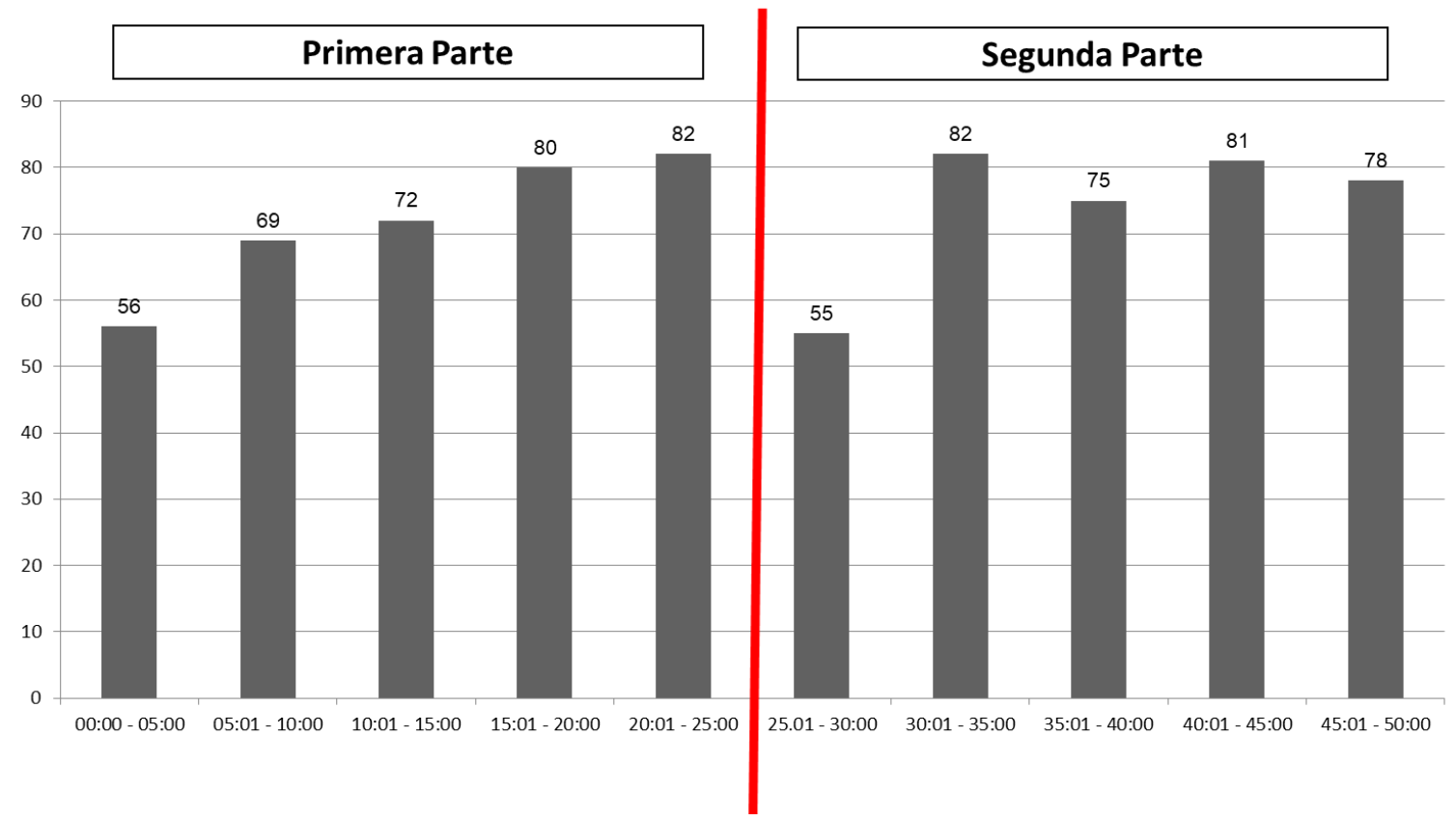

Figura 2. Franjas de tiempo de los lanzamientos a portería de los Juegos Paralímpicos de Fa5 del 2016.

En la tabla 1, se muestran el resto de resultados de las variables del estudio de Fa5.

Tabla 1. Descripción de las variables contextuales y de juego en Fa5.

\begin{tabular}{|c|c|c|c|c|c|}
\hline Variables & & & ategorías & & \\
\hline Situación lanzador & Ganador & Perdedor & Empate & & \\
\hline$n$ & 167 & 110 & 453 & & \\
\hline$\%$ & 22,9 & 15,1 & 62,1 & & \\
\hline Resultado final & Ganador & Perdedor & Empate & & \\
\hline$n$ & 342 & 200 & 188 & & \\
\hline$\%$ & 46,8 & 27,4 & 25,8 & & \\
\hline Zona de comienzo & $\begin{array}{r}\text { Zona } \\
\text { defensiva }\end{array}$ & $\begin{array}{r}\text { Zona } \\
\text { predefensiva }\end{array}$ & $\begin{array}{r}\text { Zona } \\
\text { preofensiva }\end{array}$ & Zona ofensiva & \\
\hline$n$ & 232 & 134 & 136 & 228 & \\
\hline$\%$ & 31,8 & 18,4 & 18,6 & 31,2 & \\
\hline Tipo de progresión & Combinatoria & Directa & Rápida & & \\
\hline$n$ & 104 & 283 & 343 & & \\
\hline$\%$ & 14,2 & 38,8 & 47,0 & & \\
\hline Zona lanzamiento & $\begin{array}{r}\text { Zona } \\
\text { defensiva }\end{array}$ & $\begin{array}{r}\text { Zona } \\
\text { predefensiva }\end{array}$ & $\begin{array}{r}\text { Zona } \\
\text { preofensiva }\end{array}$ & Zona ofensiva & \\
\hline$n$ & 15 & 19 & 45 & 651 & \\
\hline$\%$ & 2,1 & 2,6 & 6,2 & 89,2 & \\
\hline Situación golpeo & $\begin{array}{r}\text { Pase, control y } \\
\text { lanzamiento }\end{array}$ & $\begin{array}{r}\text { Pase y } \\
\text { lanzamiento }\end{array}$ & $\begin{array}{l}\text { Conducción y } \\
\text { lanzamiento }\end{array}$ & Otros & \\
\hline$n$ & 56 & 64 & 536 & 74 & \\
\hline$\%$ & 7,7 & 8,8 & 73,4 & 10,1 & \\
\hline Bloqueo & Sin bloqueo & $\begin{array}{r}\text { Bloqueo } \\
\text { delante } \\
\text { lanzador }\end{array}$ & $\begin{array}{r}\text { Bloqueo } \\
\text { misma altura } \\
\text { lanzador }\end{array}$ & $\begin{array}{r}\text { Bloqueo } \\
\text { detrás } \\
\text { lanzador }\end{array}$ & Otros \\
\hline
\end{tabular}


Rev.int.med.cienc.act.fís.deporte - vol. 19 - número 76 - ISSN: 1577-0354

\begin{tabular}{|c|c|c|c|c|c|}
\hline$n$ & 583 & 111 & 24 & 6 & 6 \\
\hline$\%$ & 79,9 & 15,2 & 3,3 & 0,8 & 0,8 \\
\hline $\begin{array}{l}\text { Oposición } \\
\text { lanzamiento }\end{array}$ & Sin oposición & Portero & $\begin{array}{r}\text { Oposición } \\
\text { lejana }\end{array}$ & $\begin{array}{r}\text { Oposición } \\
\text { cercana }\end{array}$ & Otros \\
\hline$n$ & 4 & 44 & 175 & 490 & 17 \\
\hline$\%$ & 0,5 & 6,0 & 24,0 & 67,1 & 2,3 \\
\hline Zona corporal & Pie derecho & Pie izquierdo & Otros & & \\
\hline$n$ & 617 & 92 & 21 & & \\
\hline$\%$ & 84,5 & 12,6 & 2,9 & & \\
\hline Tipo de golpeo & Pie interior & $\begin{array}{r}\text { Pie empeine / } \\
\text { pie puntera }\end{array}$ & Pie exterior & Pie tacón & Otros \\
\hline$n$ & 236 & 439 & 3 & 3 & 45 \\
\hline$\%$ & 32,3 & 60,6 & 0,4 & 0,4 & 6,2 \\
\hline $\begin{array}{l}\text { Resultado de } \\
\text { lanzamiento }\end{array}$ & Éxito. Gol & $\begin{array}{r}\text { Éxito. A } \\
\text { portería, no } \\
\text { hay gol y hay } \\
\text { rechace }\end{array}$ & $\begin{array}{r}\text { Fracaso. A } \\
\text { portería, no } \\
\text { hay gol y no } \\
\text { hay rechace }\end{array}$ & $\begin{array}{r}\text { Fracaso. } \\
\text { Fuera directo }\end{array}$ & Otros \\
\hline$n$ & 21 & 147 & 286 & 269 & 7 \\
\hline$\%$ & 2,9 & 20,1 & 39,2 & 36,8 & 1,0 \\
\hline
\end{tabular}

El resultado del análisis inferencial para analizar la asociación entre variables planteadas en el estudio del Fa5 en relación a la variable Resultado de lanzamiento y Resultado Recodificado $(R C)$, se muestran en la tabla 2.

Tabla 2. Relación entre la variable Resultado de lanzamiento y las variables independientes del estudio.

\begin{tabular}{|c|c|c|c|c|c|c|c|c|c|c|c|c|}
\hline \multirow[b]{2}{*}{ Variables } & \multicolumn{6}{|c|}{ Resultado de lanzamiento (RL) } & \multicolumn{6}{|c|}{ Resultado recodificado $(\mathrm{RC})$} \\
\hline & $x^{2}$ & gl. & Sig. & & $\varphi c$ & Sig. & $x^{2}$ & gl. & Sig. & & $\varphi c$ & Sig. \\
\hline Fase & 11,517 & 16 & 0,777 & & 0,063 & 0,777 & 4,704 & 4 & 0,319 & & 0,080 & 0,319 \\
\hline Equipo & 66,035 & 32 & 0,000 & & 0,151 & 0,000 & 31,359 & 8 & 0,000 & & 0,208 & 0,000 \\
\hline Tiempo & 2,365 & 36 & 0,038 & * & 0,134 & 0,038 & 7,276 & 9 & 0,608 & & 0,100 & 0,608 \\
\hline Sit. lanzador & 52,858 & 8 & 0,000 & * & 0,270 & 0,000 & 8,022 & 2 & 0,018 & * & 0,105 & 0,018 \\
\hline Resultado final & 27,604 & 8 & 0,001 & * & 0,138 & 0,001 & 15,567 & 2 & 0,000 & * & 0,146 & 0,000 \\
\hline Z. comie & 4,163 & 12 & 0,019 & & 0,105 & 0,019 & 10,353 & 3 & 0,016 & & 0,119 & 0,016 \\
\hline T. p & 865 & 8 & 0,772 & & 0,058 & 0,772 & 1,606 & 2 & 0,448 & & 0,047 & 0,448 \\
\hline Z. Ianzamiento & 17,400 & 12 & 0,135 & & 0,089 & 0,135 & 7,236 & 3 & 0,065 & & 0,100 & 0,065 \\
\hline Situación golpeo & 11,247 & 12 & 0,508 & & 0,072 & 0,508 & 4,581 & 3 & 0,205 & & 0,079 & 0,205 \\
\hline Bloqueo & 34,309 & 16 & 0,005 & & 0,109 & 0,005 & 6,399 & 4 & 0,171 & & 0,094 & 0,171 \\
\hline Op. lanzamiento & 12,121 & 12 & 0,436 & & 0,075 & 0,436 & 4,228 & 3 & 0,238 & & 0,076 & 0,238 \\
\hline Zona cor & 6,581 & 8 & 0,582 & & 0,067 & 0,582 & 0,630 & 2 & 0,730 & & 0,029 & 0,730 \\
\hline Tipo de golpeo & 43,171 & 16 & 0,000 & * & 0,122 & 0,000 & 11,964 & 4 & 0,018 & * & 0,128 & 0,018 \\
\hline
\end{tabular}

Sit. Lanzador: Situación del lanzador

Z. comienzo: Zona de comienzo

T. progresión: Tipo de progresión

Z. lanzamiento: Zona de lanzamiento

Op. Lanzamiento: Oposición en el lanzamiento

La relación entre la variable Resultado recodificado $(R C)$ y el Equipo $(x 2=31,359$; $g l=8 ; p<0,05)$, muestra un grado de asociación entre las categorías de las variables bajo $(\varphi c=0,208 ; p<0,05)$. Los resultados muestran que Brasil tiene una 
mayor probabilidad de lo esperado de que sus lanzamientos a portería sean Éxito ( $R T C=4,4 ; n=142)$, así como China ( $R T C=2,5 ; n=27)$. Por el contrario, Irán tiene mayor probabilidad de lo esperado de que sus lanzamientos a portería sean Fracaso $(R T C=2,5 ; n=69)$.

Respecto a la relación entre la variable Resultado recodificado y la variable Situación del lanzador $\left(x^{2}=8,022 ; g l=2 ; p<0,05\right)$, tiene un nivel de asociación bajo entre las categorías de las variables $(\varphi c=0,101 ; p<0,05)$. Los resultados muestran que el equipo que van ganando tiene una mayor probabilidad de lo esperado de que sus lanzamientos a portería sean Éxito $(R T C=2,6 ; n=54)$.

Las características de la vinculación entre la variable Resultado recodificado $(R C)$ y la variable Resultado final $\left(X^{2}=15,567 ; g l=2 ; p<0,05\right)$, muestra un nivel de asociación bajo entre las categorías de las variables $(\varphi c=0,146 ; p<0,05)$. Hay una mayor probabilidad de lo esperado de que los equipos ganadores durante las distintas fases del campeonato terminen sus lanzamientos a portería en Éxito $(R T C=3,9 ; n=103)$. Sin embargo, los equipos perdedores tienen mayor probabilidad de lo esperado de que sus lanzamientos a portería sean Fracaso $(R T C=2,7 ; n=166)$.

Respecto a la relación de dependencia entre la variable Resultado recodificado $(R C)$ y la variable Zona de comienzo $(x 2=10,353 ; g l=3 ; p<0,05)$. El grado de asociación entre las categorías de las variables es bajo $(\varphi c=0,119 ; p<0,05)$. Existe una mayor probabilidad de lo esperado de que las acciones de lanzamientos se inicien en la Zona ofensiva ( $R T C=2,4 ; n=168)$ y Zona preofensiva ( $R T C=2,0 ; n=114)$, cuyo resultado terminen en Éxito.

En cuanto a la relación entre la variable Resultado recodificado $(R C)$ y el Tipo de golpeo $\left(x^{2}=11,964 ; g l=4 ; p<0,05\right)$ muestra un grado de asociación entre las categorías de las variables bajo $(\varphi c=0,128 ; p<0,05)$. Los resultados muestran que los lanzamientos que se realizan con el Pie empeine / pie puntera $(R T C=2,3$; $n=291)$ y Pie exterior $(R T C=2,0 ; n=2)$, tienen una probabilidad mayor de lo esperado en terminar en Éxito. Los lanzamientos realizados con el Pie interior muestran una menor probabilidad de lo esperado de que sean Éxito $(R T C=-2,0$; $n=1)$.

En la tabla 3 se muestran los datos obtenidos hasta el segundo paso del análisis de Regresión Logística para establecer el modelo de predicción. 
Tabla 3. Variables incluidas en el modelo de Regresión logística en competición de Fa5.

\begin{tabular}{|c|c|c|c|c|c|c|}
\hline Variable / Categoría & $B$ & E.T. & Wald & gl. & Sig. & $\operatorname{Exp}(B)$ \\
\hline Zona de comienzo & & & 10,789 & 3 & 0,013 & * \\
\hline \multicolumn{7}{|l|}{ Zona defensiva (Variable referencia) } \\
\hline Zona predefensiva & 0,562 & 0,284 & 3,926 & 1 & 0,048 & 1,755 \\
\hline Zona preofensiva & 0,637 & 0,285 & 5,007 & 1 & 0,025 & 1,891 \\
\hline Zona ofensiva & $-0,090$ & 0,217 & 0,172 & 1 & 0,678 & 0,914 \\
\hline Tipo de golpeo & & & 11,709 & 4 & 0,020 & * \\
\hline \multicolumn{7}{|l|}{ Pie empeine / puntera (Variable referencia) } \\
\hline Pie interior & $-0,555$ & 0,207 & 7,172 & 1 & 0,007 & 0,574 \\
\hline Pie exterior & $-0,704$ & 1,239 & 0,323 & 1 & 0,570 & 0,495 \\
\hline Pie tacón & $-2,509$ & 1,254 & 4,002 & 1 & 0,045 & 0,081 \\
\hline Otros & 0,152 & 0,450 & 0,114 & 1 & 0,736 & 1,164 \\
\hline Constante & 1,427 & 0,209 & 46,570 & 1 & 0,000 & 4,167 \\
\hline
\end{tabular}

${ }^{*}$ significación $p<0,05$

Los resultados del Análisis de Regresión Logística muestran la existencia de dos variables con un nivel de significación óptimo $(p<0,05)$ que permite establecer un modelo de predicción de que se produzca el lanzamiento a portería en el contexto deportivo estudiado. Con respecto a la variable Zona de comienzo, en función de la zona donde se inicie la acción se puede predecir que el lanzamiento a portería irá a portería independientemente del resultado que se consiga (Wald $\left.x^{2}=10,789 ; p=0,013\right)$. Concretamente, cuando se inicia la acción del lanzamiento a portería en la Zona preofensiva, la probabilidad, Odds Ratio $(\operatorname{Exp}(B))$ de que se produzca lanzamiento es de un $189 \%\left(B=0,637\right.$; Wald $X^{2}=$ $5,007 ; p=0,025 ; \operatorname{Exp}(B)=1,891)$, respecto a si realiza la acción en la zona defensiva (Zona de referencia). En cuanto a la variable Tipo de golpeo, permite predecir el resultado de la acción (Wald $X^{2}=11,709 ; p=0,020$ ), de forma que cuando el jugador golpea el balón a portería con el pie interior disminuye en un $57 \%\left(B=-0,555 ;\right.$ Wald $\left.X^{2}=7,172 ; p=0,007 ; \operatorname{Exp}(B)=0,574\right)$. Los resultados sobre la validez del modelo predictivo se muestran en la tabla 4.

Tabla 4. Clasificación (Matriz de confusión). Modelo de predicción de la acción del lanzamiento.

\begin{tabular}{lrccc}
\hline \multirow{2}{*}{ Observado } & \multicolumn{3}{c}{ Pronosticado } \\
\cline { 3 - 5 } & & \multicolumn{2}{c}{ Resultado de lanzamiento (RC) } & \multirow{2}{*}{ \% correcto } \\
\cline { 3 - 5 } & Éxito & Fracaso & \\
\cline { 3 - 5 } Resultado de lanzamiento $(\mathrm{RC})$ & Fracaso & 244 & 67 & 59,1 \\
& & & 318 & 56,6 \\
\hline Porcentaje global & & & & 57,2 \\
\hline El valor del corte es 0,799 & &
\end{tabular}

Como muestra la tabla con los resultados de la clasificación de los casos, también denominada Matriz de confusión, el punto de corte calculado con la técnica de la curva COR, a través del índice de Youden (Fluss, Faraggi \& Reiser, 2005) se ajustó al 0,799 . De forma global el modelo clasifica correctamente un $57,2 \%$ de los casos (Sensibilidad=56,6\% y Especificidad=59,6\%). La prueba de Hosmer-Lemeshow sobre la bondad de ajuste, muestra que el estadístico $X 2=1,492(7) ; p=0,983$. Se rechaza la hipótesis nula de igualdad, asumiendo que el modelo se ajusta a los datos (Field, 2009). 


\section{DISCUSIÓN}

El objetivo general de la investigación fue analizar los lanzamientos a portería de los Juegos Paralímpicos 2016, en la modalidad de Fútbol para ciegos, con la finalidad de conocer las relaciones que condicionan la eficacia del lanzamiento a partir de las variables que se definen en un lanzamiento y predecir la eficacia del lanzamiento, encontrado que los equipos comienzan la acción en la zona preofensiva tienen más probabilidad de éxito, y aún más si lo realiza con la zona del pie empeine - puntera. Además, se encontraron relaciones entre la eficacia y las variables que se definen en cada lanzamiento, pudiendo predecirse el éxito.

En la literatura existente, los autores plantean el estudio de diversos indicadores o factores de rendimiento para dirigir el entrenamiento o los partidos. Estos modelos aplicados en los deportes colectivos permiten comprender la lógica del juego a través de los factores técnico-tácticos (Reina-Gómez \& HernándezMendo, 2012). Para ello, se suele describir variables concretas, ofensivas o defensivas (Thomas et al., 2009; Ibáñez et al., 2015; Marcelino et al., 2011; Sampaio et al., 2010). Generando investigaciones de gran calidad que permita que esta área avance con base en su metodología y dentro de la compresión general de los deportes (Drust, 2010). El conocimiento de los indicadores de rendimiento puede ayudar a determinar la estrategia y la táctica de los equipos (Petersen, Pyne, Portus, Cordy \& Dawson, 2008).

Los resultados del análisis descriptivo de los Juegos Paralímpicos de 2016, modalidad de Fa5, permiten extraer información relevante para los entrenadores. En líneas generales, es un deporte que tiene una variedad de acciones técnicotácticas bastante reducida, como consecuencia de las características de la modalidad deportiva, en la que todos los jugadores de campo tienen limitada la visión, excepto el portero. La mayor parte de los lanzamientos realizados son en la Fase clasificatoria y después en la Fase de cuarto de final como consecuencia del formato de la competición. Los lanzamientos se realizan primordialmente entre el minuto 20:01 y 25:00 de la primera parte, y en la segunda parte entre los 30:01 a 35:00. Esto es lógico, si se tiene en cuenta el cansancio, pues los equipos están desordenados y el juego se hace más rápido, con transiciones más frecuentes de un lugar a otro del campo (Tsitskaris et al., 2002). El Fa5 es un deporte que exige una importante capacidad de concentración de los jugadores para mantener el orden táctico (Suarez, 2014). El tiempo de juego, las faltas, así como las tarjetas mostradas pueden influir en el resultado final (GarcíaRubio et al., 2014; Reina-Gómez \& Hernández-Mendo, 2012), así como el ritmo de juego de los equipos, al igual que sucede en baloncesto (Gómez-Ruano et al., 2017).

La situación del equipo en el Momento del lanzamiento en el que se produce el golpeo predominan los resultados de empate. Son partidos con tanteo bajo y con gran dificultad de marcar gol como consecuencia del contacto con el rival es continúo y ocho jugadores (4 por cada equipo) disputan el balón en una cancha de dimensiones reducidas (Suárez, 2014) y los dos laterales del terreno de juego quedan configurados por unas vallas para dar continuidad al juego.

Respecto a la situación final del partido (Resultado final), el equipo que más 
lanza a portería es el que gana el partido. Estos resultados son similares a los trabajos que han analizado los Campeonatos del Mundo y destacan la importancia de los lanzamientos a portería en fútbol-11. Estos estudios reflejan por un lado que, en contextos de juego ajustados donde los dos equipos quieren ganar debido al margen reducido en el marcador, su dinámica de juego se basan en buscar transiciones rápidas y acciones próximas en el área de gol, aumentando el número de lanzamientos (Gómez-Ruano, et al., 2013), y son los equipos que mejores resultados tienen en competiciones y torneos (GarcíaRubio et al., 2017; Liu, et al., 2015). La posesión del balón es mayor para los equipos que terminan ganando, cuando van todavía perdiendo o empatando, que para los perdedores, pero no hay diferencias cuando los equipos ganadores ya dominan en el marcador (Lago, Martín-Acero \& Seirul-lo, 2007). Los desplazamientos de los jugadores en Fa5 son en zig-zag y rápidos para desequilibrar y desorganizar al equipo defensor. Por tanto, la finalización independientemente de su consecuencia, es en sí mismo un indicador dentro del juego (Reina-Gómez \& Hernández-Mendo, 2012).

En relación a la Zona de comienzo de la acción que finaliza con lanzamiento a portería, los datos muestran que las acciones comienzan tras el saque del portero hacía uno de los jugadores en la zona defensiva o a partir de jugadores que recuperan el balón en la zona ofensiva tras realizar presión. Tanto en un caso u otro, el jugador con balón será el encargado de trasladarlo hacia la zona de finalización para intentar acabar la acción golpeando hacia portería. Los lanzamientos que se producen en las zonas más cercanas a la portería o canasta son los que tienen más probabilidad de marcar gol o encestar a canasta (Prieto, Pérez \& Gómez-Ruano, 2013; Ibáñez et al., 2009) y son cuantificables. La finalización independientemente de su consecuencia, es en sí mismo un indicador de éxito dentro del juego (Gómez-Ruano, 2017).

El Tipo de progresión que realizan los jugadores principalmente son transiciones rápidas o directas. Los equipos de Fa5 tienden a buscar la zona de finalización los más rápido posible con el objetivo de terminar la acción en lanzamiento a portería. Excepcionalmente, realizan acciones combinatorias. Las transiciones rápidas son consecuencias de las características del contexto deportivo del Fa5 que condicionan la eficacia de la acción final, al igual que en otros deportes cooperativos, como es el caso de Hockey Hierba (Piñero, 2008), o del Floorball (Prieto et al., 2013), donde el lanzamiento depende de acciones técnicas previas como el pase. Los resultados demuestran que los contraataques son más eficaces que los ataques elaborados, datos similares a estudios relacionados con el fútbol convencional (Tenga et al., 2010). Por este motivo, es complicado jugar al Fa5, pues los jugadores están distribuidos por diferentes zonas del terreno de juego y sin poder ver la ubicación de sus compañeros, en un terreno de juego de reducidas dimensiones y con vallas laterales.

Con respecto a la Zona de lanzamiento donde finaliza la acción ofensiva, los datos muestran una tendencia a finalizar desde la zona ofensiva y zona preofensiva. Los lanzamientos realizados desde la zona defensiva y predefensiva son golpeos puntuales y casuales. Por este motivo, las medidas más amplias de efectividad ofensivas, tales como oportunidades de gol y tiros a gol, se usan comúnmente como alternativa a los goles marcados debido a la baja 
probabilidad de dar un resultado natural en el fútbol (Tenga et al., 2010). A través de estos parámetros, se llega a la conclusión que la cualidad de los lanzamientos tiene mayor importancia que su cantidad (Lago-Peñas et al., 2010). Los equipos técnicos deberán planificar los entrenamientos donde se trabajen transiciones rápidas de defensa-ataque, la presión alta, velocidad en los lanzamientos, así como las jugadas de estrategia en la zona de finalización. Las tareas deberán ser progresivas. Trabajar de forma general o estándar para después pasar a trabajar por turnos, primero con información auditiva, y luego, con la manipulación de la pelota (Gamonales, 2017). En una primera fase del entrenamiento se desarrollará un trabajo auditivo, continuando con ejercicios auditivos con pelota (pases y/o lanzamientos a portería), y por último, situaciones de partido o ejercicios establecidos con premisas en gran grupo (Campos et al., 2013). Por ello, es recomendable que se conozca cómo rinden los jugadores en la competición como en el entrenamiento (O`Donoghue, 2015). Por tanto, el análisis del rendimiento en Ciencias del Deporte permite conocer cómo predecir y prescribir el rendimiento de jugadores y equipos en base a las características de la propia modalidad deportiva.

En relación a la situación previa antes de golpear, los jugadores de Fa5 realizan principalmente conducción y lanzamiento. Es la acción técnico-táctica básica para trasladar el balón desde la zona defensiva o de recuperación a la zona de finalización. En el fútbol convencional, las secuencias largas de pases producen más goles por posesión que las cortas (Hughes \& Franks, 2005; Lago-Peñas et al., 2007). En Fa5, la opción menos empleadas es pase, control y lanzamiento, no hay posesión a través de pases sino posesión por medio del balón. Los jugadores en $\mathrm{Fa} 5$ realizan las siguientes acciones técnico-tácticas: temporalización, conducción, regate y lanzamiento a portería.

Respecto a la acción de bloqueo de los atacantes a la defensa del equipo rival, los jugadores realizan los ataques sin bloqueo, y los pocos existentes son bloqueo delante del lanzamiento. Los equipos juegan principalmente con acciones de transición rápidas. Por ello, no se realizan los bloqueos. Estos datos coinciden con el fútbol convencional, los contraataques son más eficaces que los ataques elaborados (Tenga et al., 2010). Sin embargo, existen diferencias relevantes con otros deportes para personas con discapacidad como el baloncesto en silla de ruedas donde predominan los bloqueos delante del lanzamiento a canasta (Molik et al., 2009).

La variable Oposición lanzamiento pone de manifiesto que los lanzamientos se han realizado principalmente con oposición cercana, después con oposición lejana, con oposición del portero, otros tipos de oposición y por último, sin oposición. Las causas que conducen a los jugadores rivales estar próximos entre sí, es que en Fa5 los jugadores se orientan por medio de un balón de cuero sonoro, ayudando a los jugadores a orientarse a través del sonido emitido, así como las indicaciones que realizan el portero, entrenador y guía en su correspondiente zona, los cuales estarán siempre coordinados en todo momento (Suarez, 2014). Además, en Fa5 es frecuente que haya jugadores dispersados en la trayectoria del balón a la portería, como consecuencia de los movimientos en zig-zag del jugador atacante, y en concreto, en el momento del lanzamiento hay jugadores en la defensa con intención de interceptar el lanzamiento al igual 
que ocurre en Baloncesto (lbáñez et al., 2009), donde la eficacia aumenta considerablemente cuando la presión defensiva es menor o nula. Por tanto, los jugadores de Fa5 se orientan a través del balón sonoro e indicaciones de los responsables por zonas de su equipo quienes tendrán que entrenar y estar capacitados para aporta una gran ventaja al equipo.

En cuanto a la zona corporal, los jugadores de Fa5 golpean principalmente con el pie derecho. El tipo de golpeo que predominan en los lanzamientos a portería es con la parte empeine / puntera, después con el interior del pie, otras zonas, seguida de la parte exterior del pie, y con el tacón. Los datos son muy similares a los estudios existentes en el fútbol convencional, que establecen que los tiros se realizan en la zona ofensiva y fueron con el empeine, puntera e interior (Álvarez et al., 2004; Lapresa et al., 2013).

Respecto a la variable Resultado de lanzamiento exponer que tan sólo hubo 21 goles de 730 lanzamientos a portería en situación de juego. Estos resultados muestran que los equipos de Fa5 de alto nivel presentan una escasa eficacia ofensiva. Los goles vienen en jugadas rápidas, pero no preceden de acciones de pases previos o de jugadas en las que participan más de dos jugadores. Caso contrario al fútbol convencional o fútbol-sala, donde la mayoría de los goles se producen en jugadas colectivas y en las que intervienen uno o dos jugadores, y según las defensas ordenadas requieren un mayor número de pases y jugadores para encontrar líneas de pases y poder llegar a la zona ofensiva (Álvarez et al., 2004; Lapresa et al., 2013; Liu et al., 2015).

En cuanto a los resultados del análisis inferencial confirmaron que la variable resultado recodificado $(R C)$ estaba relacionada con las variables planteadas para el estudio. Los datos obtenidos son similares en investigaciones relacionadas con otras modalidades deportivas de alta competición, que han basado su metodología en el análisis observacional, como lbáñez et al., (2009) en Baloncesto o Piñero (2008) en Hockey hierba. Trabajos caracterizados por analizar la relación que existe entre las variables tras definirlas en función del contexto deportivo y los objetivos planteados para la investigación.

La asociación entre la variable resultado recodificado $(R C)$ y las variables contextuales independientes Equipo, Situación del lanzador y Resultado final muestran que pueden influir en el éxito. Con respecto al rendimiento de los equipos, indicar que hay una relación significativa entre el ranking final y la eficacia en la consecución del gol. Brasil, equipo ganador del torneo, destacó por tener un mayor rendimiento ofensivo en relación con otros equipos que quedaron en las primeras posiciones del torneo. Las acciones que más se aproximan a la consecución del gol son las más realizada por los equipos ganadores (GómezRuano et al., 2013). Estos resultados son coherentes con estudios sobre otros contextos deportivos como el baloncesto (Ibáñez et al., 2009; Sampaio et al., 2010), que muestran una relación similar entre el ranking y el rendimiento deportivo. Argentina, a pesar de tener un buen rendimiento durante la competición, no se clasificó para la fase final (Brasil - Irán). La clasificación está condicionada por los partidos de las eliminatorias (Ibáñez et al., 2003), donde la derrota significa terminar el campeonato, aunque haya buenos indicadores de rendimiento. Por tanto, no todos los equipos tienen el mismo modelo de juego ni 
pretender dominar los mismos aspectos del partido (Reina-Gómez \& HernándezMendo, 2012). El resultado final de cada partido puede estar influenciado por causas como una decisión arbitral o un cambio en la alineación, que puede provocar un efecto considerable en el juego (Caballero et al., 2017; Lago-Peñas et al., 2010), así como por los requerimientos técnico-tácticos concretos del Fa5 (Morato et at., 2011), por las indicaciones claves del guía (Suarez, 2014) e incluso los viajes de desplazamientos de los equipos a las sedes de los partidos (García-Rubio et al., 2014).

La relación con la variable Situación del lanzador muestra que el equipo que va ganando en el momento de realizar el lanzamiento tiene más probabilidad de error, a diferencia de deportes de equipo de tanteo alto (baloncesto o balonmano), donde el resultado final es consecuencia del éxito en cada una de las posesiones o unidad de competición de cada equipo (Reina-Gómez \& Hernández-Mendo, 2012) y las diferentes causas que conducen al éxito o fracaso son cuantificables y tienen un efecto claro en el marcador (Álvarez et al., 2004). Por el contrario, en los deportes de tanto bajo como fútbol, la propuesta de los indicadores del rendimiento que contienen el desarrollo del juego es mucho más difícil, pues la finalización es en sí mismo un indicador de éxito dentro del juego (Reina-Gómez \& Hernández-Mendo, 2012). Esto ocurre en el caso del Fa5, que es un deporte complejo a pesar de tener acciones técnico-tácticas simples como temporalización, conducción, regate o lanzamiento a portería.

Con respecto a la relación entre la variable Resultado recodificado $(R C)$ y la variable Resultado final, los resultados muestran que los equipos que ganan el partido tienen mayor probabilidad de fracaso en el lanzamiento, a pesar de ser los equipos que más lanzan. Por tanto, no es posible justificar que un mayor número de lanzamientos sea una variable clave para explicar el resultado de los equipos. El formato de competición puede tener influencia sobre las características de los equipos ganadores. Los indicadores de rendimiento a nivel ofensivo y defensivo condicionan el resultado final de los partidos (Castellano et al., 2012; Gómez-Ruano et al., 2013).

Por otro lado, los resultados muestran que existen variables de juego (Zona de comienzo y Tipo de golpeo) que pueden influir en el rendimiento en los lanzamientos a portería. En el caso de las Zona de comienzo de la acción en competición de Fa5 de élite, la zona ofensiva puede considerarse como la zona de alta eficacia para iniciar la jugada y bajo riesgo de fracaso en el lanzamiento a portería. Los resultados son similares a algunos estudios previos en otros deportes, como es el caso del Floorball (Prieto et al., 2013) o del Baloncesto (Ibáñez et al., 2009) que muestran que los lanzamientos que se producen en las zonas próximas al punto de tanteo (canasta o portería) son los que tienen mayor probabilidad de éxito. Esto es consecuencia de iniciar las acciones en la zona defensiva, tras el saque del portero hacía uno de los jugadores, que pierde el balón en la zona de peligro, pues los jugadores rivales recuperan la posesión del balón en la zona ofensiva tras realizar presión. Los entrenadores deberán planificar ejercicios donde se trabaje la presión alta, así como salidas de balón para evitar las perdidas en la zona de peligro siempre respetando el reglamento. Los lanzamientos a portería son realizados con el pie derecho y concretamente con la zona del empeine - puntera. Por ello, existe una mayor probabilidad de lo 
esperado de conseguir éxito, puesto que este tipo de lanzamiento se suele utilizar en disparos cercanos o lejanos. Estos datos corroboran los resultados de estudios existentes en fútbol-sala (Álvarez et al., 2004; Lapresa et al., 2013).

Los resultados del análisis predictivo, al igual que en otras modalidades deportivas colectivas como el baloncesto (Ibáñez et al., 2009) o el fútbol (Taylor, Mellalieu, James \& Shearer, 2008), permiten predecir la aparición del gol en Fa5 en situación de juego. Es un modelo predictivo simple, dando lugar a un deporte accesible para personas con deficiencia visual o ciegas. Se puede afirmar que, en Fa5 de élite, iniciar la acción previa al lanzamiento en las zonas próximas de finalización (Zona ofensiva y preofensiva) aumenta de manera considerable la probabilidad de marcar gol. Además, ejecutar el lanzamiento desde las zonas alejadas disminuye la probabilidad de marcar gol, así como utilizar distintos tipos de golpeo diferentes al empeine-puntera.

\section{CONCLUSIÓN}

El Fa5 es un deporte de invasión de tanteo bajo con particularidades específicas, que lo diferencian del resto de modalidades de fútbol en las acciones técnicotácticas que realizan los jugadores, tales como los tipos de progresión, los bloqueos, o táctica de juego, que influye de forma directa en los lanzamientos a portería en situación de juego. La forma de juego que ha permitido un mayor éxito en el campeonato analizado ha sido la recuperación del balón en zona preofensiva, con una conducción rápida hacia la portería contraria, con un lanzamiento fuerte con la puntera/empeine.

El resultado del lanzamiento está condicionado por las acciones que lo preceden y definen la forma de juego más eficaz en esta modalidad deportiva en cada competición, en función de los equipos participantes y del sistema de competición. Los equipos muestran diferentes modelos de juego. El efecto de marcar en primer lugar incrementa las probabilidades de éxito en los posteriores lanzamientos a portería. Llevar la iniciativa en el marcador predecirá el resultado final del partido.

Los entrenadores de Fa5 deben diseñar tareas de entrenamiento en la que se apliquen las secuencias de juego eficaces, así como situaciones defensivas que favorezcan rápidas recuperaciones del balón en la zonas próximas a portería y finalicen con progresiones directas.

\section{REFERENCIA BIBLIOGRÁFICAS}

Agresti, A., \& Finlay, B. (2009). Statistical methods for the social sciences $\left(4^{\mathrm{a}}\right.$ ed.). San Francisco: Pearson.

Álvarez, J., Puente, J., Manero, J., \& Manonelles, P. (2004). Análisis de las acciones ofensivas que acaban en gol de la liga profesional de fútbol sala española. Revista de Entrenamiento Deportivo, 4, 27-32.

Caballero, P., García-Rubio, J., \& Ibáñez, S. J. (2017). Análisis de la influencia de las variables situacionales en el rendimiento en fútbol U'18. Retos, 
Nuevas Tendencias en Educación Física, Deporte y Recreación, 32, 224227.

Campos, L. F., Borin, J. P., Nightingale, T., Silva, A. A., Araújo, P. F., \& Gorla, J. I. (2014). Alterations of Cardiorespiratory and Motor Profile of Paralympic 5a-side Football Athletes during 14-Week In-Season Training. International Journal of Sports Science, 4(6A), 85-90. https://doi.org/10.5923/s.sports.201401.12

Campos, L. F., De Athayde, A., Dos Santos, L., Costa, L. T., Montagner, P., Borin, J., De Araújo, P., \& Gorla, J. (2013). Effects of training in physical fitness and body composition of the Brazilian 5-a-side football team. Revista Andaluza de Medicina del Deporte, 6(3), 91-95. https://doi.org/10.1016/S18887546(13)70041-8

Castellano, J., Casamichana, D., \& Lago-Peñas, C. (2012). The use of match statistics that discriminate between successfut and unsuccessfun soccer teams. Journal of Human Kinetics, 31, 139-147. https://doi.org/10.2478/v10078-012-0015-7

Crewson, P (2006). Applied statistics handbook. AcaStat Software, Leesburg.

Drust, B. (2010). Performance analysis research: meeting the challenge. Journal of Sport Sciences, 28(9), 921-922. https://doi.org/10.1080/02640411003740769

Field, A. (2009). Discovering statistics using SPSS ( $3^{a}$ ed.). London: Sage Publications Ltd.

Fluss, R., Faraggi, D., \& Reiser, B. (2005). Estimation of the youden index and its associated cutoff point. Biometrical Journal, 47(4), 458-472. https://doi.org/10.1002/bimj.200410135

Gamonales, J.M. (2017). Fútbol a 5 para personas ciegas como contenido de Educación Física. Revista Profesional de Investigación, Docencia y Recursos Didácticos, 80(3), 66-70.

Gamonales, J.M., León, K., Muñoz, J., González-Espinosa, S., \& Ibáñez, S. J. (2018a). Validación del IOLF5C para la eficacia del lanzamiento en fútbol para ciegos. Revista Internacional de Medicina y Ciencias del Deporte, 18(70), 359-379. http://dx.doi.org/10.15366/rimcafd2018.70.010

Gamonales, J.M., Muñoz, J., León, K., \& Ibáñez, S. J. (2018b). Entrenamiento y confiabilidad entre observadores en el análisis del fútbol para ciegos. Retos, Nuevas Tendencias en Educación Física, Deporte y Recreación, 34(2), 155161.

García-Rubio, J., Gómez-Ruano, M. A., Lago-Peñas, C., \& Ibáñez, S. J. (2017). Effect of match venue, scoring first and quality of opposition on match outcome in the UEFFA Champions League. International Journal of Performance Analysis in Sport, 15, 527-539. https://doi.org/10.1080/24748668.2015.11868811

García-Rubio, J., Ibáñez, S. J., Gómez-Ruano, M. A., \& Sampaio, J. (2014). Basketball Game-related stastics discriminating ACB league outcome and final score differences. International Journal of Performance Analysis in Sport, 14, 443-452. https://doi.org/10.1080/24748668.2014.11868733

Gómez-Ruano, M. A. (2017). La importancia del análisis notacional como tópico emergente en Ciencias del Deporte. RICYDE, Revista Internacional de Ciencias del Deporte, 13(47), 1-4. https://doi.org/10.5232/ricyde2017.047ed 
Gómez-Ruano, M. A., Gómez-López, M.T., \& Jiménez-Sáiz, S.L. (2013). Diferencias entre equipos ganadores y perdedores de fútbol en función del tipo de partido mediante el estudio de indicadores de rendimiento. Revista Euroamericana de Ciencias del Deporte, 2(1), 37-41. http://dx.doi.org/10.6018/185731

Gómez-Ruano, M.A., Ibáñez, S.J., Parejo, I., \& Furley, P. (2017). The use of classification and regression tree when classifying winning and losing basketball teams. Kinesiology, 49(1), 47-56. https://doi.org/10.26582/k.49.1.9

Gorla, J. I., De Athayde, A., De Campos, L. Dos Santos, C. F., de Almeida, J. J. G., Duarte, E., \& Queiroga, M. R. (2017). Composição corporal e perfil somatotípico de atletas da seleção brasileira de futebol de 5. Revista Brasileira de Ciências do Esporte, 39(1), 79-84. https://doi.org/10.1016/j.rbce.2015.12.016

Hernández, J. (2005). Análisis de las estructuras del juego deportivo (3 ed.). Zaragoza: INDE.

Hughes, M. D., \& Bartlett, R. M. (2002). The use of performance indicators in performance analysis. Journal of Sports Sciences, 20(10), 739-754. https://doi.org/10.1080/026404102320675602

Hughes, M. D., \& Franks, I. M. (2005). Analysis of passing sequences, shots and goals in soccer. Journal of Sports Sciences, 23(5), 509-514. https://doi.org/10.1080/02640410410001716779

Ibáñez, S. J., Feu, S., García-Rubio, J., Parejo, I., \& Cañadas, M. (2009). Shot diferences between professional (ACB) and amateur (EBA) basketball teams. Multifactorial study. Revista de Psicología del Deporte, 18, 313-317.

Ibáñez, S. J., Santos, J. A., \& García-Rubio, J. (2015). Multifactorial analysis or free throw shooting in eliminatory basketball games. International Journal of Performance Analysis in Sport, 15, 897-912. https://doi.org/10.1080/24748668.2015.11868839

Ibáñez, S.J., Sampaio, J., Sáenz-López, P., Giménez, J., \& Janeira, M. A. (2003). Game statistics discriminating the final outcome of junior World Basketball Championship Matches (Portugal 1999). Journal of Human Movement Studies, 45, 1-19.

Lago-Peñas, C., Lago-Ballestero, J., Dellal, A., \& Gómez-Ruano, M. (2010). Game related statistics discriminated winning, drawing and losing teams from the Spanish soccer league. Journal of Sports Science and Medicine, 9, 288293.

Lago-Peñas, C., Martín-Acero, R., \& Seirul-lo, F. (2007). El rendimiento en el fútbol. Una modelización para el F.C. Barcelona. Apunts, 90, 51-58.

Lapresa, D., Álvarez, L., Arana, J., Garzón, B., \& Caballero, V. (2013). Observational analysis of the offensive sequences that ended in a shot by the winning team of the 2010 UEFA Futsal Championship. Journal of Sports Sciences, 31(15), 1731-1739. https://doi.org/10.1080/02640414.2013.803584

Liu, H., Gómez-Ruano, M.A., Lago-Peñas, C., \& Sampaio, J. (2015). Match statistics related to winning in the group stage of 2014 Brazil FIFA World Cup. Journal of Sports https://doi.org/10.1080/02640414.2015.1022578 
Magno, M. P., Morato, M. P., Bilzon, J. L., \& Duarte, E. (2013). Sports injuries in Brazilian blind footballers. International Journal of Sports Medicine, 34(3), 239-243. https://doi.org/10.1055/s-0032-1316358

Marcelino, R., Mesquita, I. \& Sampaio, J. (2011). Effects of quality of opposition and match status on technical and tactical performances in elite volleyball. Journal of Sports Sciences, 29(7), 733-741. https://doi.org/10.1080/02640414.2011.552516

McGarry, T. (2009). Applied and theoretical perspectives of performance analysis in sport: Scientific issues and challenges. International Journal of Performance Analysis in Sport, 9(1), 128-140. https://doi.org/10.1080/24748668.2009.11868469

Menard, S. (2002). Applied logistic regression analysis. California: Sage Publications.

Molik, B., Kosmol, A., Morgulec-Adamowicz, N., Laskin, J. J., Jezior, T., \& Patrzatek, M. (2009). Game efficiency of elite female wheelchair basketball players during World championships (Gold Cup) 2006. European Journal of Adapted Psysical Activity, 2(2), 26-38.

Montero, I., \& León, O. G. (2007). A guide for naming research studies in Psychology. International Journal of Clinical and Health psychology, 7(3), 847-862.

Morato, M. P., Gomes, M. S. P., Duarte, E., \& De Almeida, J. J. G. (2011). A leitura de jogo no futebol para cegos. Movimento (ESEF/UFRGS), 17(3), 97114.

Newell, J., Aitchison, T., \& Grant, S. (2014). Statistics for sports and exercise science: a practical approach. Routledge.

O'Donoghue, P. (2010). Research methods for sports performance analysis. London and New York: Routledge Taylor \& Francis Group.

O`Donoghue, P. (2015). An introduction to performance analysis of sport. London and New York: Routledge Taylor \& Francis Group.

Petersen, C., Pyne, D., Portus, M.R., Cordy, J., \& Dawson, B. (2008). Analysis of performance at the 2007 Cricket World Cup. International Journal of $\begin{array}{llll}\text { Performance Analysis in } & \text { 8port, } 1 \text {, }\end{array}$ https://doi.org/10.1080/24748668.2008.11868417

Piñero, R. (2008). Observación y análisis de la acción de gol en hockey hierba. Sevilla: Wanceulen.

Prieto, M., Pérez, J., \& Gómez-Ruano, M. A. (2013). Indicadores de rendimiento ofensivo en el floorball de alto nivel. Revista Internacional de Ciencias del Deporte, 9(32), 114-125. https://doi.org/10.5232/ricyde2013.03202

Rein, R., \& Memmert, D. (2016). Big data and tactical analysis in elite soccer: future challenges and opportunities for Science. Springer Plus, 5(1), 1410. https://doi.org/10.1186/s40064-016-3108-2

Reina-Gómez, A., \& Hernández-Mendo, A. (2012). Revisión de indicadores de rendimiento en fútbol. Revista Iberoamericana de Ciencias de la Actividad Física y el Deporte, 1(1), 1-14.

Reina-Gómez, A., Hernández-Mendo, A., \& Fernández-García, J.C. (2010). Multi-facet design for goal scoring in soccer-7. Quality and Quantity, 44(5), 1025-1035. https://doi.org/10.1007/s11135-009-9253-8

Sampaio, J., Lago-Peñas, C., Casais, L., \& Leite, N. (2010). Effects of starting score-line, game location, and quality of opposition in basketball quarter 
score. European Journal of Sport Science, 10(6), 391-396. https://doi.org/10.1080/17461391003699104

Suarez, G. (2014). Importancia del rol del guía o llamador en el fútbol para ciegos. Revista electrónica de Ciencias Aplicadas al Deporte, 7(25).

Taylor, J.B., Mellalieu, S.D., James, N., \& Shearer, D.A. (2008). The influence of match location, quality of opposition, and match status on technical performance in professional association football. Journal of Sport Sciences, 26(9), 885-895. https://doi.org/10.1080/02640410701836887

Tenga, A., Ronglan, L.T., \& Bahr, R. (2010). Measuring the effectiveness of offensive match-play professional soccer. European Journal of Sport Science, 10(4), 269. https://doi.org/10.1080/17461390903515170

Thomas, C., Fellingham, G., \& Vehrs, P. (2009). Development of a notational analysis system for selected soccer skills of a Women's college team. Measurement in Physical Education and Exercise Science, 13, 108-121. https://doi.org/10.1080/10913670902812770

Tsitskaris, G., Theoharopoulos, A., Galanis, D., \& Nikopoulou, M. (2002). Types of shots used at the Greek National Basketball Championship according to the división and position of players. Journal of Human Movement Studies, 42, 43-52.

Valez, A., Areces, A., Blanco, H., \& Arce, C. (2011). Diseño y aplicación de una batería multidimensional de indicadores de rendimiento para evaluar la prestación competitiva en el fútbol de alto nivel. International Journal of Sport Science, 7(23), 103-112. https://doi.org/10.5232/ricyde2011.02303

Velten, M. C., Bläsing, B., Portes, L., Hermann, T., \& Schack, T. (2014). Cognitive representation of auditory space in blind football experts. Psychology of Sport and Exercise, 15(5), 441-445. https://doi.org/10.1016/j.psychsport.2014.04.010

Velten, M. C., Ugrinowitsch, H., Portes, L. L., Hermann, T., \& Bläsing, B. (2016). Auditory spatial concepts in blind football experts. Psychology of Sport and Exercise, 22, 218-228. https://doi.org/10.1016/j.psychsport.2015.08.010

Referencias totales / Total references: 57 (100\%) Referencias propias de la revista /Journal's own references: $2(3,50 \%)$

Rev.int.med.cienc.act.fís.deporte - vol. 19 - número 76 - ISSN: 1577-0354 